\title{
Mental Health in Spouses of Iraq-Iran War Veterans With PTSD
}

\author{
Arsia Taghva ${ }^{1}$, Parviz Dabbaghi ${ }^{2}$, Susan Shafighi ${ }^{3}$, Seyyed Mohammad Ali Mortazaviha ${ }^{3}$, \\ Vahid Donyavi ${ }^{1, *}$ \\ ${ }^{1}$ Department of Psychiatry, Aja University of Medical Sciences, Tehran, IR Iran \\ ${ }^{2}$ Department of Clinical Psychology, Aja University of Medical Sciences, Tehran, IR Iran \\ ${ }^{3}$ Faculty of Nursing, Aja University of Medical Sciences, Tehran, IR Iran \\ *Corresponding author: Vahid Donyavi, Department of Psychiatry, Aja University of Medical Sciences, Tehran, IR Iran. Tel/Fax: +98-2122481806, E-mail: donyavi_vahid@yahoo.com.
}

Received: December 19, 2013; Revised: December 21, 2013; Accepted: December 24, 2013

\begin{abstract}
Background: Mental challenges are among the most frequent complications of wars, afflicting individuals directly or indirectly involved in military confrontations. Post-traumatic stress disorder(PTSD) is one of the most common disorders in such situations.

Objectives: Our goal is to assess mental health of spouses of Iraq-Iran war veterans with post-traumatic stress disorder(PTSD).

Materials and Methods: In this cross-sectional study, two groups of the spouses of the veterans, 40 cases diagnosed with PTSD as the case group and 40 cases who were not, as the control group, were enrolled into the study and compared. Mental health was assessed using the general health questionnaire (GHQ-28) which has four major domains including somatic symptoms, anxiety and insomnia, depression, and social malfunction.

Results: Twenty-two patients (55\%) in case group and 11 patients (27.5\%) in control group had impaired general health status ( $\mathrm{P}<0.05, \mathrm{P}$ value $=0.02)$. Nineteen patients $(47.5 \%)$ in case group and 9 patients $(22.5 \%)$ in control group complained of somatic symptoms $(\mathrm{P}$ value $=$ $0.001)$. Anxiety and insomnia (45\% vs. 22.5\%) (P value $=0.002)$ and depression $(47.5 \%$ vs. $25 \%)(\mathrm{P}$ value $=0.008)$ were more common in the case group.

Conclusions: Although imposed Iran-Iraq war ended 25 years ago, we observed that spouses of veterans with PTSD have more somatic complaints and poorer mental health compared to spouses of veterans without PTSD. Further studies seem essential regarding proper management and provision of psychology consultation services along with medical treatments to raise mental health of their spouses.
\end{abstract}

Keywords: Stress Disorders, Post-Traumatic; Veteran; War; Spouses; Questionnaires

\section{Background}

Mental challenges are among the most frequent complications of wars, afflicting individuals directly or indirectly involved in military confrontations. Physical injuries usually result in disabilities, while mental injuries will afflict the victims often long after the war event. This damage is imposed not only on people who have experienced the event, but also will involve their families (1).

Post-traumatic stress disorder (PTSD) is one of the most common disorders in such situations. It constitutes a type of stress disorder resulting from the experience of an event in which the person has encountered true death, threat of death or serious injuries for others or him/herself. The disorder manifests as repeated and irritating re-experiences of the event in the form of thoughts, fantasies or perceptions. The lifelong prevalence of PTSD is estimated about $8 \%$ in general population, $10-12 \%$ in women and 5-6\% in men (2).

PTSD is a considerable psychiatric condition among soldier veterans who participated in Iraq-Iran war (19801988). A study evaluated psychiatric victims of the war in
Qom province, Iran, in 2001, using medical records and face-to-face interview, concluding that more than $80 \%$ were diagnosed with PTSD with their symptoms ranging from mild to very severe (3). In another study conducted by Nourbala et al. on 400 psychiatric cases of wartime veterans, $34.6 \%$ were found to have neurotic disorders (anxiety, depression and adjustment disorders) and 27.6\% were diagnosed with PTSD (4).

Results from previous studies indicate that family members of psychiatric patients are at considerable risk of tension (5). Moreover, caring for a chronic patient causes distress and hardship for caregivers, thus influencing their quality of life (6). The findings of a qualitative study on spouses of patients suffering from psychiatric disorders indicated that these difficult and chronic responsibilities will profoundly compromise the quality of life and satisfaction of spouses living with patients (7).

Clinical experiences and repeated observations of researchers suggest that families of veterans may experience hardships to a greater extent than the victims them-

Implication for health policy/practice/research/medical education:

Iran-Iraq war ended about 25 years ago but its sequences never did. This survey assessed the psychological impacts of war on spouses of veterans with post-traumatic stress disorder (PTSD).

Copyright (C) 2014,Aja University of Medical Sciences. This is an open-access article distributed under the terms of the Creative Commons Attribution License, which permits unrestricted use, distribution, and reproduction in any medium, provided the original work is properly cited. 
selves. Calhoun et al. conducted a study to conclude that the severity of symptoms and interpersonal violence in PTSD patients is associated with the mental pressure felt by caregivers (8). Spouses of PTSD patients face greater challenges for coping with and tolerating the personal characteristics of PTSD (including mood, aggression, stubbornness and dominance) and feel a lower level of satisfaction of their marital relationship (including sharing of feelings and opinions, information exchange, comfort and compatibility) (9).

It appears that with war termination and combatants returning home, the stress caused by the war and its detrimental impact on the combatants will continue to comprise their lives and their families' health, especially their spouses.

\section{Objectives}

As mentioned above, previous studies have detected a considerable frequency of PTSD among Iranian veterans. A few studies, however, were found to have addressed the issue of psychiatric disorders among family members of PTSD patients, especially their spouses. Thus, we undertook the present study to investigate this subject in order to contribute to better care for PTSD victims and their spouses.

\section{Materials and Methods}

In this cross- sectional study, we selected and compared the two groups of 40 women, comprising of the spouses of Iraq-Iran war veterans. The case group contained 40 women whose husbands were diagnosed with PTSD. The control group consisted of 40 women whose husbands served in the war but did not have PTSD. Anyone with known psychiatric disorder was excluded from the control group. The women were referred to the psychiatric clinic of 505 Iranian Army Hospital, Tehran, Iran as companions to their husbands during routine medical visits in 2009.

A diagnosis of PTSD had been previously established for the patients in case group, at least by 2 psychiatrists and these patients had medical records in the psychiatric clinic. Since the study subjects were military personnel who had served in the war and their history indicated the onset of their condition to coincide with the war, their PTSD was deemed war related. The control group did not reveal any signs in their psychological examinations and were not diagnosed with any kinds of psychiatric disorders. Many of the participants had gotten married during the war. The questionnaires were anonymous and the participants were informed that the information will be used strictly for research purposes and will not have any bearing on their official status. Oral consents were taken from all of the participants and the protocol was approved by Committee of Medical Ethics in Psychiatric and Psychological Department in addition to Medical Ethics Center of Aja University of Medical Sciences, Tehran, Iran.

The general health questionnaire-28 (GHQ-28) (10) translated by Palahang (11), was used to evaluate the participants' mental health. The questionnaire consists of 4 scales (somatic symptoms, anxiety and insomnia, social malfunction, and depression), each was assessed with 7 questions. The scoring is accomplished with a simple Likert's scale, ranking the answer options from 1 to 4 . Summing up the answers of each scale yields the health score for that scale. The total sum of all 28 questions provides an assessment of each individual's mental health.

Numerous studies have indicated a high validity for the GHQ. The reliability of the questionnaire was assessed by Taghavi to be 90\% (12) and the Cronbach's alpha values were $84 \%$ for physical symptoms, $79 \%$ for social functioning, 81\% for depression and 91.5\% for mental health (13).

The data were analyzed by the SPSS software (ver. 16.0) and comparisons between the two groups were made by chi-squared analysis for variables of the GHQ-28 and the t-test for ages.

\section{Results}

The two groups were not significantly different in terms of mean age. The mean ages of the case and control groups were $42.9 \pm 3.7$ and $43 \pm 3.8$ years, respectively ( $\mathrm{P}>$ 0.05). The frequency of disorders was significantly higher in the case group compared to the control group in all scales of somatic symptoms, anxiety and insomnia, social malfunction, and depression as well as the general health status (Table 1 ).

Table 1. Comparing the Items of the GHQ-28 Between Partners of Military Personnel With PTSD and Without PTSD

\begin{tabular}{llll}
\hline & Case Group, No. (\%) & Control Group, No. (\%) & P value \\
\hline Disorder of general health & $22(55)$ & $11(27.5)$ & 0.02 \\
Somatic symptoms & $19(47.5)$ & $9(22.5)$ & 0.001 \\
Anxiety and insomnia & $18(45)$ & $9(22.5)$ & 0.002 \\
Depression & $19(47.5)$ & $10(25)$ & 0.008 \\
Social malfunction & $20(50)$ & $11(27.5)$ & 0.001 \\
\hline
\end{tabular}




\section{Discussion}

Similar to its previous counterparts, the present study also indicates that psychiatric disorders are more frequent among family members of patients with PTSD. Caring for patients with chronic disorders exerts pressure and tension on the caregivers, compromising their quality of life. Recent studies, such as one conducted by Dezhkam (14), indicate that the difficult and constant responsibilities in routine life profoundly lower quality of life and satisfaction with living with one's spouse.

Families of PTSD patients are concerned about their compatibility and emotional and sexual issues. Physical violence against spouses and children is not rare among PTSD patients, and their wives often suffer doubly, both from their husbands' behavior and the pressure to protect their children against these behaviors (14). Other studies have pointed out that physical and verbal abuse is more common in families with PTSD victims. The abuse is most often directed at the spouse and the spouses of PTSD patients are at higher risk of anger, which will exacerbate the marital incompatibility (1). Some studies concluded that Vietnam veterans diagnosed with PTSD experience less satisfaction with their marital relationships. Additionally, half of their spouses felt that nothing can prevent the failure of their marriage (1).

PTSD is a disorder which affects combatants' quality of life, as well as that of his family and society. Repeated observations and clinical experience of researchers indicate that families of these victims suffer to a greater extent. Calhoun et al. (8) conducted a study to conclude that the severity of symptoms and interpersonal violence in PTSD patients is associated with greater pressure felt by caregivers.

One of many negative aspects of wars is the large number of survivors with psychiatric complications. According to the statistics published in 1999 by the Iranian Society of Veterans, there are 36,354 victims with documented psychiatric disorders in Iran (15). The findings of the present study, indicating the impacts of war-related PTSD on mental health of these patients' spouses (increased anxiety, depression, somatic symptoms, and social malfunction) compared to the spouses of the normal individuals, corroborated the results of previous studies from different countries.

In conclusion, based on our findings, we recommend future studies to be directed at identifying the factors affecting general health in spouses of military personnel with PTSD, in order to provide knowledge into ways of controlling these risk factors and thus improve general health in this population.

Although Iraq-Iran war ended many years ago, it appears that the veterans and their families are still at risk of psychiatric disorders. Therefore, we propose a plan of preventive measures against PTSD in family members, prioritizing fulfillment of their basic needs such as hous- ing, employment, financial security, and access to medical care for veterans and their families. Furthermore, considering the impacts of war on spouses and children of veterans, we recommend the establishment of adjustment facilities (similar to the other countries which have experienced war) to provide counseling and professional services for veterans in order to help them re-adjust with their families and society, and for controlling their anxiety and stress management and coping with daily challenges using self-help groups would be helpful. It would be better if we had access to duration of the veterans' war attendance, and knew the exact time of their marriage before or after war.

\section{Acknowledgements}

It was our honor to work with some memorial groups of the Great War. Through, we would like to appreciate their unforgettable efforts and participation in the present study.

\section{Authors' Contribution}

All authors had substantial contribution in data gathering, drafting article, and interpretation of data.

\section{Financial Disclosure}

We had no financial interests related to the material in the manuscript.

\section{Funding/Support}

There was no support.

\section{References}

1. Mastsakis A. Vietneam wires: facing the challenges of life with veterans suffering PTSD.Brooklandville: Sidran press; 1996.

2. Kaplan HI, Sadock BJ. Kaplan and Sadock's synopsis of psychiatry: Behavioral sciences, clinical psychiatry. 9th ed. London: Mosby; 2007.

3. Borhani H. Assessing Mental Health in Families of Psychiatric War Victims in Qom Province. Tehran: Shahid Beheshti University;2001.

4. Noorbala AA. [Psychiatric disorder in combatants]. J Mil Med. 2002;4(4):257-60.

5. Martens L, Addington J. The psychological well-being of family members of individuals with schizophrenia. Soc Psychiatry Psychiatr Epidemiol. 2001;36(3):128-33.

6. Sales E. Family burden and quality of life. Qual Life Res. 2003;12(Suppl 1):33-41.

7. Jungbauer J, Wittmund B, Dietrich S, Angermeyer MC. The disregarded caregivers: subjective burden in spouses of schizophrenia patients. Schizophr Bull. 2004;30(3):665-75.

8. Calhoun PS, Beckham JC, Bosworth HB. Caregiver burden and psychological distress in partners of veterans with chronic posttraumatic stress disorder.J Trauma Stress. 2002;15(3):205-12.

9. Akbari MA. World Health Report 2001. Tehran: Ebne Sina Cultural Institute; 2003.

10. Goldberg DP, Hillier VF. A scaled version of the General Health Questionnaire. Psychol Med.1979;9(1):139-45.

11. Palahang H, Nasr M, Barahani MT, Shah Mohammadi D. [Epidemiology of mental liiness in Kashan city]. Andisheh va Raftar J. 2003;2:19-27.

12. Taghavi MR. Assessing the Reliability and Validity of General Health Questionnaire. J Educ Psychol. 2001;4:381-98. 
13. Torkesaluye SG, Mehdipoor A, Fatemi R, Azmsha T. Mental Health Assessment of Volunteers Entering the Academic Field of Physical Educatio. Middle-East J Sci Res. 2013;13(2):203-6.

14. Comparing the Mental Health between partners of war-related psychiatric victims referring to Sadr and Imam Hosein Hospi- tals. In: Dezhkam M, Aminorroaya A editors. 1st scientific conference on war victims and families. 2003 Tehran.

15. Sirati M. Evaluating the personal, familial, social and economical factors affect the recurrence of mental disorders from the veterans' point of view. Baghiatallah University of Medical Sciences; 2002. 